\title{
A Study on User Perception of Personality-Based Recommender Systems
}

\author{
Rong $\mathrm{Hu}$ and Pearl $\mathrm{Pu}$ \\ Human Computer Interaction Group \\ Swiss Federal Institute of Technology in Lausanne (EPFL) \\ CH-1015, Lausanne, Switzerland \\ \{rong.hu,pearl.pu\}@epfl.ch
}

\begin{abstract}
Our previous research indicates that using personality quizzes is a viable and promising way to build user profiles to recommend entertainment products. Based on these findings, our current research further investigates the feasibility of using personality quizzes to build user profiles not only for an active user but also his or her friends. We first propose a general method that infers users' music preferences in terms of their personalities. Our in-depth user studies show that while active users perceive the recommended items to be more accurate for their friends, they enjoy more using personality quiz based recommenders for finding items for themselves. Additionally, we explore if domain knowledge has an influence on users' perception of the system. We found that novice users, who are less knowledgeable about music, generally appreciated more personalitybased recommenders. Finally, we propose some design issues for recommender systems using personality quizzes.
\end{abstract}

Keywords: Recommender System, Personality, Domain Knowledge, User Study, User Modeling.

\section{Introduction}

Recently, researchers suggested that personality characteristics can be used to build user profiles in recommender systems, inspired by the findings in psychological studies [8]. Studies have indicated that there is a significant connection between personality and people's tastes and interests. For example, Rentfrow and Gosling [14] revealed that musical preferences are associated with individual differences in personality, ability and self-perception. Moreover, due to their ability of lessening the cold start problem associated with commonly adopted collaborative filtering recommender systems, personality-based recommender systems are increasingly attracting the attention of researchers and industry practitioners [5].

Based on different recommendation recipients, recommender systems can be classified into two groups: one suggests items to the active users; the other predicts items for the people who don't directly interact with the system. The former has been studied widely in the literature 11. The latter is known as gift finders that help users identify ideal gift for others, such as their friends [3].

P. De Bra, A. Kobsa, and D. Chin (Eds.): UMAP 2010, LNCS 6075, pp. 291 3022010.

(C) Springer-Verlag Berlin Heidelberg 2010 
The absence of recipients' interaction with systems makes recommendation more difficult, since the information which can be acquired to predict recipients' preferences is not as sufficient as that for active users. To resolve this issue, intelligent gift finders were developed based on recipients' personal characteristics, such as gender, age, occasion, life styles, and personalities [3]. Even though an increasing number of well-known online shopping websites, e.g., Yahoo shopping, Ebay.com, have tried to incorporate intelligent gift finders into their systems, the subject remains an open area of study in this field. Due to the important role of gift finders in commercial websites, it is believable that the related studies could greatly benefit the e-commerce society.

Our previous research showed that using personality quizzes is a viable and promising way to build users' profiles for the recommendation of entertainment products [8]. The tested personality quiz based system was preferred to a baseline rating-based system mainly due to its prominent merit on ease of use. In the present study, we are trying to gauge the values of using personality quizzes to build profiles not only for an active user but also his or her friends as a personality-based gift finder. On the other hand, prior research has revealed that prior domain knowledge is a crucial attribute which influences the way in which users interact with a system and further impacts their perceptions [11, 12. We therefore are wondering whether the prior domain knowledge have an influence on users' perception to the personality-based recommender system in both cases.

In this paper, we first proposed a general personality-based recommendation method in music domain on the basis of the results reported in [14. To our knowledge, no previous work has explicitly dealt with this issue in the literature. Then, an in-depth within-subject user study was conducted to compare two ways of building user profiles using personality quizzes in recommender systems: for active users and for their friends. We further explored the influence of domain knowledge on user perceptions of the tested personality-based recommender system in both cases. The evaluation criteria include objective ratings of the recommended songs and subjective measurements on perceived usefulness, perceived ease of use, attitudes and behavior intentions [4. The results reveal several designing issues for recommender systems using personality quizzes.

The remainder of this paper is organized as follows. In Sect. 2 we present background and related work; then we describe the method of user modeling and musical preference inference framework in Sect. 3. And we describe our user study in detail on experiment setup, hypotheses, produce and design, experiment results and discussion in Sect. 4 followed by conclusion and future work.

\section{Background and Related Work}

\subsection{Personality-Based Recommender Systems}

According to Burger 2], personality is defined as a "consistent behavior pattern and intrapersonal processes originating within the individual". It is relatively stable and predictable. Studies also show that personalities influence human decision making process and interests [9, 14. Drawing on the inherent 
inter-related patterns between users' personalities and their interests/behaviors, personality-based recommenders are designed to provide personalized services. Lin and Mcleod [10] proposed a temperament-based filtering model incorporating human factors, especially human temperaments (Keirsey's theory), into the processing of an information recommendation service. Their model categorizes the information space into 32 temperament segments. Combining with the contentbased filtering technique, their method aims at recommending the information units which best matched both users' temperaments and interests. Even though the system utilizes personalities to model user profiles, they don't really take the psychological relation between human personalities and information items into account. In [13], authors applied the relation between musical preferences and personality traits found in [14 to recommend music. However, they didn't explain how it works in detail. In the current work, we propose a general algorithm for personality-based music recommender systems on the basis of the results reported in [14]. It can be easily generalized to other domains.

\subsection{Personality and Musical Preferences}

In the landmark work 14, according to the statistical results on a large-scale dataset, Rentfrow and Gosling found four musical preferences groups: reflective and complex(e.g., blues, jazz, classical and folk); intense and rebellious (e.g., rock, alternative, and heavy metal music); upbeat and conventional (e.g., country, religious, and pop music); and energetic and rhythmic (e.g., rap/hip-hop, soul/funk, and electronic/dance music). Most importantly, they empirically revealed that the four musical preferences are not only associated with the level of complexity, emotionality and energy of musical compositions, but also individual differences in personality, ability and self-perception.

More specifically, the fascinating pattern of links between musical preferences and personality (Big-Five model was used to measure personality in the work) revealed from the correlation analysis showed that the reflective and complex dimension is positively related to Openness to New Experience; the intense and rebellious dimension was positively related to Openness to New Experiences; the upbeat and conventional dimension has positive correlations with Extraversion, Agreeableness and Conscientiousness, and a negative correlation with Openness to New Experience; the energetic and rhythmic dimension is positively related to Extraversion and Agreeableness. More explanations can refer to [14].

\subsection{Influence of Prior Domain Knowledge}

Research has shown that prior domain knowledge is a critical attribute which influences the way in which users interact with a system and further impacts their perceptions [6, 11, 12. It has been revealed that domain expertise enhances search performance. Expert users with a higher level of domain knowledge tend to find information in a more flexible and efficient way [11, 12]. However, domain novice users rely more on the simple searching functions of the informationseeking tools systems provide. On the other hand, research has indicated that 
domain novice users can get more help on reinforcing their knowledge when using information systems [12. Contrastingly, domain expert users require more advanced information to satisfy their advanced needs. We are trying to investigate its influences in recommender systems.

\section{User Modeling and Musical Preference Inference}

Generally, the recommendation problem can be formalized as follows [1. Let $U$ be the set of all users and let $P$ be the set of items or categories to be recommended (In our application, it is the set of musical preference dimensions). $P F$ is defined as a prediction function that measures the possibility of one item $p$ is liked by user $u$, i.e., $P F: U \times P \rightarrow R$, where $R$ is a totally ordered set (e.g., nonnegative integers or real numbers within a certain range). Then for each user $u \in U$, we want to choose such item $p_{u}^{\prime} \in P$ that maximizes the inferred preference value. More formally,

$$
\forall u \in U, p_{u}^{\prime}=\operatorname{argmax}_{p \in P} P F(u, p) .
$$

In the following, we propose a general algorithm framework for inferring liked musical preference in terms of user personalities. The possibility of one musical dimension $\mathrm{p}$ is liked by user $\mathrm{u}$ is predicted by considering two factors: the personality of user $\mathrm{u}$ and the relations between the personality and four musical preference dimensions. More specifically, we present personality characteristics described in the Big-Five model as a vector $\mathbf{p s}_{\mathbf{u}}=\left(p s_{u}^{o}, p s_{u}^{c}, p s_{u}^{e}, p s_{u}^{a}, p s_{u}^{n}\right)^{T}$ for user $u$. Here, $p s_{u}^{o}, p s_{u}^{c}, p s_{u}^{e}, p s_{u}^{a}$ and $p s_{u}^{n}$ represent the values in the dimension of openness to new experience, conscientiousness, extraversion, agreeableness and neuroticism respectively. Their values are normalized to the range $[-1,1]$. The user preference model is described as $\mathbf{m p}_{\mathbf{u}}=\left(m p_{u}^{r c}, m p_{u}^{i r}, m p_{u}^{u c}, m p_{u}^{e r}\right)^{T}$, where, $m p_{u}^{r c}, m p_{u}^{i r}, m p_{u}^{u c}$ and $m p_{u}^{e r}$ represent the extent to which the user u like reflective and complex, intense and rebellious, upbeat and conventional, and energetic and rhythmic music respectively. Therefore, user preference model $\mathbf{m p}_{\mathbf{u}}$ can be calculated as,

$$
\mathbf{m p}_{\mathbf{u}}=W \times \mathbf{p s}_{\mathbf{u}}
$$

where,

$$
W=\left[\begin{array}{ccc}
w_{11} & \cdots & w_{15} \\
\vdots & \ddots & \vdots \\
w_{41} & \cdots & w_{45}
\end{array}\right] .
$$

$W$ is a 4-by-5 weighting matrix. The value of $w_{i j}$ means the strength of the relation between personality trait $j$ and musical preference dimension $i$, and $w_{i j}$ is also normalized in the range of $[-1,1]$. The positive value represents a positive relationship between a personality trait and a musical preference dimension. That is, a user have a higher value in the personality trait $j$, will like the music preference dimension i with a higher possibility, vice versa. On the other hand, the 
negative $w_{i j}$ indicates a negative relationship between personality trait $j$ and musical preference dimension $i$. The magnitude of $w_{i j}$ represents the strength of such relations. The larger is this value, the stronger dominates the personality trait $j$ on the musical preference dimension $i$. In our prototype system, we assign $w_{i j}$ with the correlation value between music preference dimension $i$ and personality trait $j$ reported in Rentfrow.

Then, the musical preference dimension $m p_{u}^{k}, k \in\{r c, i r, u c, e r\}$, with the maximal value is the one which user $u$ might most like. As an alternative to increase recommendation diversity, the musical preference dimensions whose corresponding values in $\mathbf{m p}_{\mathbf{u}}$ are more than a defined threshold $r$ are picked out and the number of recommended songs in these dimensions are in proportion to the predicted preference values. In our study, we adopted the second strategy.

\section{Experiment: User Study}

We conducted a user study aiming to compare two ways of using personality quizzes to build user profiles in recommender systems, with the help of an music recommender implemented based on the algorithm described above. We also investigated the influence of domain knowledge on user perceptions of recommender systems in both cases.

\subsection{Hypotheses}

As mentioned above, it is greatly difficult to select an item as gift, since the recipient's preferences are often not well known. We assume that the personality-based recommendation technology using personality quizzes to build profiles is more appreciated by users when it is used to suggest songs as gifts, compared to the situation when it is used to recommend songs for users themselves. Additionally, as we can imagine, if one user is an expert on music, he or she could easily choose songs to listen to. Contrastively, it is reasonable to assume that domain novice users with a low level of knowledge on music can get more help from our system. Therefore, they would give higher evaluation scores. On the other hand, we assume expert users can also perceive the usefulness when they try to find songs for their friends, considering the lack of knowing well about their friends' preferences on music. We therefore formulate the following two hypotheses:

H1: The personality quiz based music recommender system is perceived more positively when it is used to recommend music for friends compared to when it is used to recommend music for users themselves.

H2: Users with low level of music knowledge perceive more usefulness of the system when they use personality quizzes to find songs for both themselves and their friends, compared to those with high level of music knowledge.

H3: Users with high level of music knowledge can also perceive the usefulness of the system when they use personality quizzes to find songs for their friends. 


\subsection{Experiment Setup}

We prepared 1,581 songs (1956 - 2009) covering all 14 genres in the four musical preferences. We assigned genre labels to all songs by consulting several popular music websites (last.fm, new.music.yahoo.com, and itunes.com). After finding out user preferred musical preferences using the method described in the previous section, in each preferred musical preference dimension, recommended songs were selected evenly from all included genres and randomly in each genre.

TIPI (Ten Item Personality Inventory) [7] was used to assess users' personalities (Big Five Personality Traits) on a 7-point scale ranging from 1 (strongly disagree) to 7 (strongly agree). The acquisition process takes about a few minutes.

\subsection{Experiment Design and Procedure}

This user study was conducted in a within-subject design. All subjects were required to use personality quizzes to find songs for themselves and also for one of their friends as gifts. To minimize the carryover effects (both practice and fatigue effects), all subjects were randomly assigned to two experimental conditions. Each condition had a different order in usage scenarios. That is, half the subjects in one condition tried to use personality quizzes to find songs for themselves first and then for one of their friends. The other condition had the reverse sequence. To avoid any possible confusion during evaluation, subjects were told that they would evaluate two systems. One only recommends songs for users themselves and the other for friends.

The user study was launched online. An online procedure containing instructions, evaluated systems and post-study questionnaires was implemented so that participants could follow easily. In the first page of the online user study, participants were debriefed on the objective of the experiment and the upcoming tasks, and then they started the evaluation. The main user tasks include:

1. Answer a list of background question, such as gender, music knowledge etc.

2. Accomplish the TIPI for self/friend.

3. Listen to 20 recommended songs. If these songs are suggested to themselves, subjects are asked to rate them on a 5-point scale from 1 (dislike it very much) to 5 (like it very much). Otherwise, they are asked to send this list to the friend whom these songs are found for. It can be easily done with one function implemented in our online procedure.

4. Fill in a post-questionnaire on subjective perceptions of the evaluated system. Each question is to be answered on a 5-point Likert scale ranging from 1 (strongly disagree) to 5 (strongly agree).

Two versions of post-study questionnaire were designed for two usage scenarios, and they cover 10 measurements. All questions are listed in Table 1. If the questions on one measurement use the same sentence for two scenarios, only one is presented. 
Table 1. Subjective evaluation questions (building profile for active users/ for friends)

\begin{tabular}{|c|c|}
\hline Measurements & Questions \\
\hline $\begin{array}{l}\text { perceived } \\
\text { effectiveness }\end{array}$ & $\begin{array}{l}\text { This website was effective in recommending songs for me. / This } \\
\text { website was effective in recommending songs for my friend. }\end{array}$ \\
\hline $\begin{array}{l}\text { perceived } \\
\text { accuracy }\end{array}$ & $\begin{array}{l}\text { The songs suggested to me corresponded to my taste. / I was } \\
\text { confident that the songs suggested to my friend correspond to } \\
\text { his(her) taste. }\end{array}$ \\
\hline $\begin{array}{l}\text { perceived } \\
\text { helpfulness }\end{array}$ & $\begin{array}{l}\text { The website helped me discover music for myself. / The website } \\
\text { helped me to discover music for my friends. }\end{array}$ \\
\hline enjoyment & $\begin{array}{l}\text { I enjoyed using personality quizzes to get recommendations. / I } \\
\text { enjoyed using personality quizzes to find songs for my friends. }\end{array}$ \\
\hline ease to use & I found this site easy to use. \\
\hline satisfaction & I am satisfied with the overall functions of this website. \\
\hline use intention & $\begin{array}{l}\text { If this were a real website, I would use it to get music recom- } \\
\text { mendations. / If this were a real website, I would use it to find } \\
\text { songs for my friends. }\end{array}$ \\
\hline $\begin{array}{l}\text { purchase } \\
\text { intention }\end{array}$ & $\begin{array}{l}\text { If necessary, I would buy the recommended songs. / If necessary, } \\
\text { I would buy the recommended songs for my friends as gift. }\end{array}$ \\
\hline return intention & I will use this type of recommender systems again. \\
\hline $\begin{array}{l}\text { reference } \\
\text { intention }\end{array}$ & I will introduce this website to my friends \\
\hline
\end{tabular}

\subsection{Participants}

A total of 80 subjects (32 females) were recruited in our user study. Most of them (69 out of 80) are students at a university in Switzerland and others work in the related fields, including software engineers, designers, music promoters and graphic designers. All subjects listen to music frequently. To make the group as diverse as possible, the subjects were selected from a variety of nationalities (17 countries) and varying levels of music knowledge. The distribution of users' domain knowledge levels is described in the experiment results section. 55 subjects have the experience of using music recommenders. As the reward, all subjects were told to have a chance to win one novel generation iPod Shuffle (4G) valued at $99 \mathrm{CHF}$ in a final lottery draw.

\section{Results and Discussion}

\subsection{Objective Measure}

The objective measure aims at evaluating the recommendation quality. All participants were asked to rate the 20 songs recommended for themselves on a 5 -point scale from 1 (dislike it very much) to 5 (like it very much). On the other hand, participants were encouraged to send a "gift" with 20 songs to the friends 
whom they found these songs for, and to ask their friends to rate these "gift" songs on the same scale. Eventually, 21 "friends" accomplished the rating task.

The results show that there is no significant difference between the ratings to the songs recommended to active users themselves and those to the songs recommended to friends (Independent t-test: $\mathrm{t}=1.369, \mathrm{p}=0.171$ ). Regarding recommendations based on profiles built by users themselves, on average, 14.14 out of 20 songs are rated as acceptable (rated higher than "it's ok"), and wherein 8.29 songs are considered to be liked. For recommendations based on profiles built by friends, 14.57 out of 20 songs are acceptable, and 9.09 songs are thought to be liked.

\subsection{Subjective Measure}

The average scores of users' responses to the subjective measurements are shown in Fig. 1. Paired t-test was conducted to find significant differences. As we can see, subjects scored significantly higher on perceived accuracy when finding songs for friends than for themselves (mean $=2.8, \mathrm{SD}=0.925$ vs. mean $=3.1, \mathrm{SD}=$ 0.941 , respectively; $\mathrm{t}=-2.287, \mathrm{p}=0.025)$. Subjects also gave significantly higher scores on enjoying using personality quizzes to find songs for themselves than for friends $($ mean $=3.6, \mathrm{SD}=0.951$ vs. mean $=3.2, \mathrm{SD}=1.178$, respectively; $\mathrm{t}=$ $3.001, \mathrm{p}=0.004)$, and they are both higher than the median value (3.0). Subjects scored significantly higher on return the system to find songs for themselves than for friends (mean $=3.2, \mathrm{SD}=0.986$ vs. mean $=3.0, \mathrm{SD}=1.012$, respectively; $\mathrm{p}=0.047)$.

The results show a peak in the perceived ease of use in both scenarios, which indicates that participants strongly felt that this personality quiz-based system is easy to use. However, there is no significant difference between two scenarios $($ mean $=4.3, \mathrm{SD}=0.89$ for finding songs for self vs. mean $=4.25, \mathrm{SD}=0.89$ for finding songs for friends; $\mathrm{t}=0.49, \mathrm{p}=0.626$ ).

As for the helpfulness of the personality-based music recommender, surprisingly, the difference between finding songs for users themselves and for friends is not significant by paired T-test $($ mean $=3.1, \mathrm{SD}=1.105$ vs. mean $=2.9$, $\mathrm{SD}=0.917$, respectively; $\mathrm{t}=1.454, \mathrm{p}=0.15)$. Similarly, the comparisons on satisfaction (both are slightly higher than 3.5 ) and behavior intentions show no significant difference.

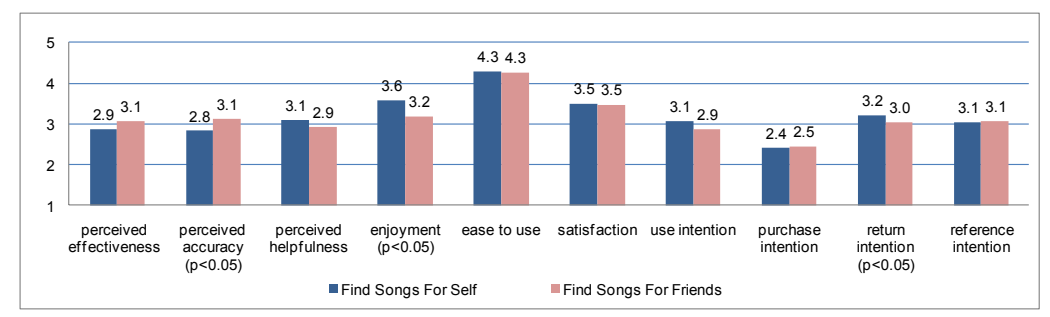

Fig. 1. Subjective evaluation comparison between finding songs for self and for friends 


\subsection{Influence of Domain Knowledge}

Among all participants, 17 subjects strongly agreed that they have knowledge about music (expert users), 32 subjects agreed (medium users), 23 subjects had a neutral opinion (novice users) and 8 subjects didn't think they have any prior knowledge about music. According to [6], users do not have a well defined perception until they reach some degree of expertise. Additionally, only 8 out of 80 subjects thought they didn't have any knowledge of music. Their responses are not sufficient for statistical analysis. We therefore decided to eliminate their responses from our analysis. One-way ANOVA was conducted with prior knowledge levels as IVs and subjective scores as DVs. Then, we conducted post-hoc pairwise comparisons (Bonferroni) to identify how the three levels of prior knowledge varied from one another on the measurements with overall significant differences among three levels.

Figure 2 shows the mean scores of subjective measurements in terms of three different levels of music knowledge given the scenario of finding songs for themselves. The ANOVA results indicate significant differences in participants' perceived effectiveness $(\mathrm{F}(2,69)=7.173, \mathrm{p}=0.001)$, perceived accuracy $(\mathrm{F}(2,69)$ $=3.147, \mathrm{p}=0.049)$, perceived helpfulness $(\mathrm{F}(2,69)=6.333, \mathrm{p}=0.003)$ and use intention $(\mathrm{F}(2,69)=5.273, \mathrm{p}=0.007)$. Pairwise comparison results show that medium users scored significantly higher than expert users on perceived effectiveness (mean: 3.34 , SD: 0.827 vs. mean: 2.29 , SD: 1.105 , respectively; $\mathrm{p}=0.001$ ), perceived accuracy (mean: 3.03, SD: 0.933 vs. mean: 2.35 , SD: 0.996, respectively; $\mathrm{p}=0.044$ ), perceived helpfulness (mean: 3.34, SD: 1.035 vs. mean: 2.29, SD: 0.985, respectively; $\mathrm{p}=0.004$ ) and use intention (mean: 3.31 , SD: 1.061 vs. mean: 2.35 , SD: 1.057 , respectively; $\mathrm{p}=0.308$ ). In addition, we found that novice users scored significantly higher than expert users on perceived helpfulness (mean: 3.30, SD: 1.105 vs. mean: 2.29 , SD: 0.985 , respectively; $\mathrm{p}=0.011$ ) and use intention (mean: 3.21 , SD: 0.951 vs. mean: 2.35 , SD: 1.057 , respectively; $\mathrm{p}=0.031$ ).

Figure 3 shows the mean scores of subjective measurements in terms of three different music knowledge levels given the scenario of finding songs for friends. The ANOVA result indicate significant differences in participants' perceived effectiveness scores $(\mathrm{F}(2,69)=3.490, \mathrm{p}=0.036)$ and return intention $(\mathrm{F}(2,69)$ $=3.617, \mathrm{p}=0.032$ ). Pairwise comparison results show that novice users scored significantly higher than expert users (mean: 3.43, SD: 0.843 vs. mean: 2.59,

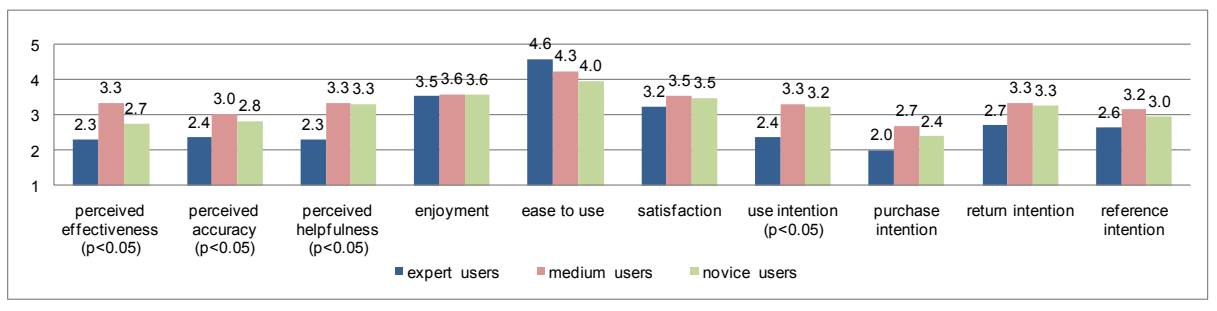

Fig. 2. Subjective evaluation comparison among different music knowledge levels in the scenario of finding songs for self 


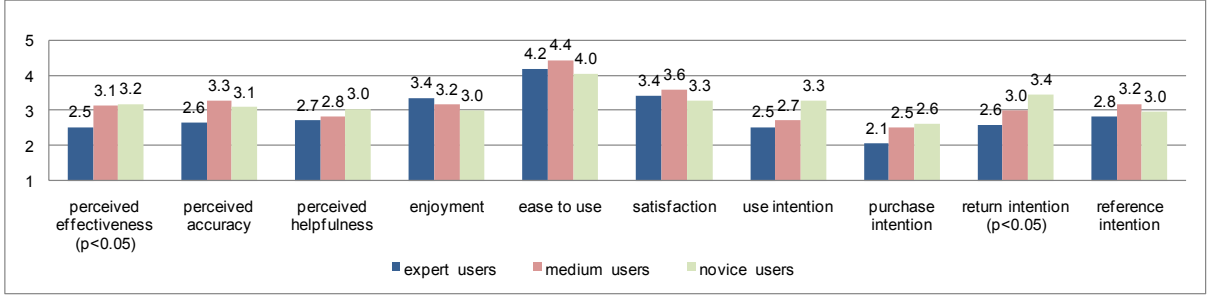

Fig. 3. Subjective evaluation comparison among different music knowledge levels in the scenarios of finding songs for friends

SD: 0.870 , respectively; $\mathrm{p}=0.028$ ) on return intention. However, no significant differences are found between each two of knowledge levels on perceived effective, although the overall comparison is significantly different among all levels of domain knowledge. Furthermore, we found that domain expert users scored moderately higher on perceived helpfulness when using the system to find songs for friends than doing it for themselves (paired t-test: mean $=2.71, \mathrm{SD}=0.686$ vs. mean: 2.29 , SD: 0.985 , respectively; $\mathrm{p}=0.090)$.

\subsection{Discussion}

From the results of objective measure, we could see that in both cases, about $3 / 4$ of the recommended songs are rated as acceptable and half of them are rated as to be liked. There is no significant difference between two compared scenarios. Regarding the subjective evaluation, participants, in general, expressed that users enjoyed using personality quizzes to get recommendations and satisfied with the overall functions in both cases. The finding that the personality quiz-based recommender systems were strongly perceived to be easy to use was revalidated in the present study. It is surprising to see that, while active users perceive the recommended items to be more accurate for their friends, they enjoy more using the system to find songs for themselves. It doesn't support our hypothesis $H 1$ that personality-based technology is more appreciated by users when they try to find songs for friends.

It can be seen that the scores on behavior intentions (intention to use the system, purchase the recommended songs, return to the system and introduce to friends) are low. It suggests some challenges of the acceptance of personalitybased recommender systems, especially when they are used as gift finders. One important obstacle is privacy issues. As stated in [13, users were really worried about how their personal information can be used and whom this private information will be shown to. Similar phenomena happened in our experiment. Furthermore, when they were asked to use personality quizzes to find songs for friends, some participants expressed that they were worried about that their counterpart friends would see how they evaluated them, especially on some sensitive measurements, such as neuroticism. These might explain why the scores were pushed down on behavior intentions. This sheds light on design issues on how to reduce such negative effects inherent to personality-based recommender 
systems. For example, it might be a possible solution to design interfaces with explanations to tell who will see this personal information and what it will be used for, provide more benefits to users in order to trade off the fear of the risk from disclosing personal information [13].

The results also show that prior domain knowledge do influence users' perception of the system. Domain novice and medium users had significantly more positive perceptions than expert users, and further higher intention to use and return to the system. On the other hand, domain expert users scored moderately higher on perceived helpfulness when using the system to find songs for friends than for themselves. Therefore, our hypothesis $H 2$ is perfectly supported and $\mathrm{H} 3$ is somehow sustained. It is indicated that the way of using personality quizzes to build user profiles cannot satisfy the advanced needs of domain expert users. There is a need to design adaptive personality-based recommender systems. For example, for expert users, we could integrate other modeling methods to increase user control, e.g., leveraging rating-based methods to update user preference models [1]. For novice users, systems with simple operating interfaces and low requirements on domain knowledge could be more helpful.

\section{Conclusion and Future Work}

With the help of an implemented personality-based music recommender system based on the general algorithm framework we proposed in this paper, an indepth within subject user study was conducted to investigate user perceptions of two ways of using personality quizzes to build user profiles, for an active user and for his or her friends. We further explored the influences of domain knowledge to the user perception of the system. The results show that users enjoyed using personality quizzes to get recommendations and satisfied with the overall functions in both cases. However, while active users perceive the recommended items to be more accurate for their friends, they enjoy more using the system to find songs for themselves. On the other hand, users with low level of music domain knowledge scored more higher on perceived usefulness than domain expert users. However, expert users perceived slightly more helpful when trying to find songs for friends. These results shed light on several design issues for personality-based recommender systems. As the further work, it is worth comparing the personality-based modeling method with other existed modeling technologies, such as collaborative filtering and content-based methods.

Acknowledgments. We thank the EPFL and Chinese Government for sponsoring the reported research work. We are grateful to the participants for their patience and time.

\section{References}

1. Adomavicius, G., Tuzhilin, A.: Toward the Next Generation of Recommender Systems: A Survey of the State-of-the-Art and Possible Extensions. IEEE Trans. Knowledge and Data Eng. 17(6), 734-749 (2005) 
2. Burger, J.M.: Personality, 7th edn. Thomson/Wadsworth, Belmont (2008)

3. Chan, F.Y., Cheung, W.K.: A Knowledge-based Recommender System for Customized Online Shopping. In: Proceedings of the World Congress on Mass Customization and Personalization 2001, MCPC'01. HKUST, TUM, Hongkong (2001)

4. Davis, F.D., Bagozzi, R.P., Warshaw, P.R.: User acceptance of computer technology: A comparison of two theoretical models. Management Science 35(8), 982-1003 (1989)

5. Dunn, G., Wiersema, J., Ham, J., Aroyo, L.: Evaluating Interface Variants on Personality Acquisition for Recommender Systems. In: Houben, G.-J., McCalla, G., Pianesi, F., Zancanaro, M. (eds.) UMAP 2009. LNCS, vol. 5535, pp. 259-270. Springer, Heidelberg (2009)

6. Frias-Martinez, E., Chen, S.Y., Macredie, R.D., Liu, X.: The role of human factors in stereotyping behavior and perception of digital library users: a robust clustering approach. Journal of User Modeling and User-Adapted Interaction 17(3), 305-337 (2007)

7. Gosling, S.D., Rentfrow, P.J., Swann Jr., W.B.: A very brief measure of the BigFive personality domains. Journal of Research in Personality 37, 504-528 (2003)

8. $\mathrm{Hu}, \mathrm{R} ., \mathrm{Pu}, \mathrm{P} .: \mathrm{A}$ comparative user study on rating vs. personality quiz based preference elicitation methods. In: Proceedings of the 13th international conference on Intelligent User Interfaces, pp. 367-372 (2009)

9. Jung, C.: Psychological Types. Princeton, New Jersey (1971)

10. Lin, C., McLeod, D.: Temperament-Based Information Filtering: A Human Factors Approach to Information Recommendation. In: Proc. of the IEEE International Conference on Multimedia and Exposition, vol. 2, pp. 941-944 (2000)

11. Lazonder, A.W., Biemans, H.J.A., Wopereis, I.G.J.H.: Differences between novice and experienced users in searching information on the World Wide Web. Journal of the American Society for Information Science 51(6), 576-581 (2000)

12. Mitchell, T.J.F., Chen, S.Y., Macredie, R.D.: Hypermedia learning and prior knowledge: Domain expertise vs. system expertise. Journal of Computer Assisted Learning 21(1), 53-64 (2005)

13. Perik, E.M., Ruyter, B., de Markopoulos, P., Eggen, J.H.: The Sensitivities of User Profile Information in Music Recommender Systems. In: Proceedings of Private, Security, Trust, pp. 137-141 (2004)

14. Rentfrow, P.J., Gosling, S.D.: The do re mi's of everyday life: The Structure and Personality Correlates of Music Preferences. Journal of Personality and Social Psychology 84, 1236-1256 (2003) 